Théologiques

Théologiques

\title{
La théologie des religions de Claude Geffré
}

\section{Wasim Salman}

Volume 17, numéro 2, 2009

Querelles d'images?

URI : https://id.erudit.org/iderudit/044070ar

DOI : https://doi.org/10.7202/044070ar

Aller au sommaire du numéro

\section{Éditeur(s)}

Faculté de théologie et de sciences des religions, Université de Montréal

\section{ISSN}

1188-7109 (imprimé)

1492-1413 (numérique)

Découvrir la revue

\section{Citer cet article}

Salman, W. (2009). La théologie des religions de Claude Geffré. Théologiques, 17(2), 225-244. https://doi.org/10.7202/044070ar

\section{Résumé de l'article}

L'article expose la théologie des religions de Claude Geffré en la situant dans son contexte de théologie catholique des religions. De Babel à Pentecôte est l'ouvrage dans lequel le théologien français se rend compte de la situation pluraliste du monde. Il s'agit d'une approche théologique qui propose des solutions et fait une lecture créative de la tradition chrétienne, notamment de celle des semences $d u$ Verbe. Nous retenons certains points essentiels de la théologie de Geffré : le caractère kénotique du christianisme et la réflexion sur la signification du pluralisme. 


\section{La théologie des religions de Claude Geffré}

Wasim SALMAN"

Dans le cadre de notre recherche sur le rapport entre herméneutique philosophique et théologie, nous avons été amené à approfondir l'importante contribution théologique de Claude Geffré et à étudier comment ce théologien dominicain opère un tournant décisif dans l'histoire de la théologie catholique, lorsqu'il inscrit la compréhension théologique dans le cercle herméneutique gadamérien. L'affirmation la plus incisive du tournant herméneutique qu'il opère est "qu'il n'y pas de théologie en dehors d'une inscription dans l'histoire et dans la culture» (Geffré 2006a, 27). Ainsi, la théologie est de bout en bout une entreprise herméneutique.

C'est lui, le premier théologien français en date, à avoir intégré la tradition herméneutique dans le concept catholique de théologie hérité de Thomas d'Aquin. Geffré insiste sur la corrélation entre l'expérience chrétienne primitive et l'expérience humaine présente, alors que l'usage des catégories aristotéliciennes en théologie avait transformé la compréhension de l'intellectus fidei en une perspective dogmatique qui transmettait la tradition, sans procéder à aucune réflexion sur ce qu'elle implique.

La théologie herméneutique s'appuie sur une compréhension partant d'une fusion d'horizons avec mise en relation du passé et du présent ${ }^{1}$. Elle prend au sérieux le rapport entre l'expérience de la communauté primitive et l'expérience actuelle du troisième millénaire, dont le pluralisme religieux

* Wasim Salman est docteur en théologie de l'Université pontificale grégorienne de Rome. Ses recherches actuelles portent sur le rapport entre Gadamer et Bultmann: histoire et démythologisation, ainsi que sur l'impact de la théologie dialectique dans la philosophie herméneutique de Gadamer et la pensée théologique de ce dernier. Il a récemment publié (2010), La Wirkungsgeschichte de Hans-Georg Gadamer dans la théologie de Claude Geffré, David Tracy et Wolfhart Pannenberg, préface par C. Geffré, Rome, Grégorienne.

1. Sur la fusion des horizons, voir Gadamer (1986, 311); aussi Grondin (2005, 401-418).

(C) Revue Théologiques 2009. Tout droit réservé. 
est un phénomène majeur. Si la théologie herméneutique vise à établir la communication de l'expérience de la communauté primitive avec celle de la communauté actuelle, la théologie interreligieuse tâche de conceptualiser l'échange des témoignages, en vue de la communication d'expériences religieuses.

Dans cette recherche, nous aborderons la question du pluralisme religieux tel qu'il est traité par C. Geffré dans ses derniers ouvrages, à savoir: Croire et interpréter (2001a) et De Babel à Pentecôte (2006a). Nous parcourrons le développement historique de la théologie des religions en y situant Geffré, puis nous établirons le rapport entre kénose et théologie interreligieuse. Nous traiterons finalement de la signification du pluralisme religieux dans le dessein salvifique de Dieu.

\section{Développement historique de la théologie des religions}

Partons d'une triple distinction entre exclusivisme, inclusivisme et pluralisme (Richard 2002, 27; aussi Dupuis 2002, 123). Le premier courant affirme qu'entre le christianisme et les autres religions, il n'y a pas d'équivalence possible - les religions, pures créations humaines, n'expriment qu'un simple geste vers Dieu, alors que la révélation chrétienne relève d'un geste de Dieu envers l'humain (Dupré La Tour 1999, 218). Aussi, seul le christianisme enraciné dans la révélation est-il porteur du salut advenu en Jésus-Christ. Cet exclusivisme ecclésiologique, dominant jusqu'au XX siècle, se condense dans cet adage: Extra ecclesiam nulla salus ( Hors de l'Église point de salut »; Gibellini 2008, 156).

Le deuxième courant a pour représentants J. Daniélou (1948; 1953), H. de Lubac (1946), Y. Congar (2000) et K. Rahner (1965, 545-554; 1983, 179-202), qui ont développé, au cours de la période pré-conciliaire, la "théorie de l'accomplissement», permettant de qualifier les religions non chrétiennes de "pierres d'attente» ou de préparations évangéliques au christianisme (Dupuis 1997, 201).

J. Daniélou, premier théologien à avoir développé une théologie de l'accomplissement, distingue, quant à lui, entre religion et révélation, de sorte que seule la révélation judéo-chrétienne relève de l'ordre du surnaturel².

2. Pour approfondir la théologie de J. Daniélou, nous renvoyons le lecteur à ses œuvres; voir aussi Guggenheim (2006, 240-257). 
C'est à $\mathrm{H}$. de Lubac et à K. Rahner que nous devons le dépassement du dualisme entre révélation naturelle et Révélation surnaturelle. En maintenant le christocentrisme, ils soutiennent que les autres traditions religieuses peuvent être porteuses de valeurs salutaires. Ainsi, H. de Lubac parle du «désir naturel du surnaturel» (1965), tandis que K. Rahner développe la théorie des chrétiens anonymes, reposant sur l'orientation de l'homme vers Dieu, en vertu de son «existential surnaturel» $\left(1983,154^{3}\right)$.

S'interrogeant sur la vérité des cultures asiatiques que le christianisme n'a pu atteindre et sur la restriction du christianisme dans son territoire qu'est l'Occident, Rahner pousse à ses extrêmes la théologie de l'accomplissement et discerne dans les religions la présence agissante du mystère de Jésus-Christ (Dupuis 2002, 89). Puisque, en effet, les religions sont une expression de l'orientation originelle et surnaturelle de tout homme vers le Christ, les non-chrétiens sont sauvés nécessairement par le Christ (Sesboüé 1984, 521-535). Sans doute Dieu veut-il offrir dans son Fils le salut à tous les hommes et les atteindre par sa grâce, même au-delà des frontières visibles de l'Église. Appréciant ainsi les valeurs positives des religions mondiales, ces théologiens ont voulu dépasser l'exclusivisme ecclésiologique en faveur d'un inclusivisme christologique.

Rahner distingue entre histoire catégoriale de la révélation et histoire transcendantale du salut: de fait, l'histoire du salut qui culmine en JésusChrist est coextensive à l'histoire du monde (1983, 180). Sans doute Dieu s'est-il révélé par les paroles des prophètes, mais aussi par des paroles de sagesse à l'extérieur de l'histoire d'Israël. Et de là découle une compréhension différente de l'histoire des religions, qui n'est plus alors la simple histoire de l'agir religieux de l'homme, mais l'œuvre du même Dieu de l'Ancien et du Nouveau Testament - sans nier pour autant les ambiguités et la perversion des phénomènes religieux. Rahner pose la Révélation de JésusChrist comme le critère de discernement d'une quelconque révélation divine ou déviation humaine.

Rahner étend le dessein salvifique à l'histoire universelle dont «l'histoire de la Révélation vétérotestamentaire de l'Alliance procède» (1983,

3. Rahner définit l'autocommunication de Dieu comme suit : «le mouvement transcendantal de l'esprit, ordonné au mystère absolu dans la connaissance et la liberté, est porté par Dieu lui-même en son autocommunication de telle sorte que ce mouvement a son terme et son origine, non pas dans le mystère sacré comme but éternellement lointain et qui ne serait jamais atteignable qu'asymptotiquement, mais dans le Dieu d'absolues proximité et immédiateté ». 
202). L'homme libre est toujours mis à la disposition du mystère absolu, à travers les formes extérieures de rites et de vie relationnelle. Il s'ensuit que toute l'histoire de la Révélation trouve son aboutissement dans la personne du Christ, parce que l'homme, quelle que soit son appartenance religieuse, ne cesse de réfléchir sur la Révélation et sur sa relation transcendantale avec Dieu. L'histoire de la Révélation biblique est un moment particulier de l'histoire universelle du salut et de la révélation qui atteint son point culminant dans l'autocommunication de Dieu aux hommes dans l'Incarnation de Jésus-Christ.

L'Église n'est pas une société fermée, exclusiviste: le Dieu infini agit hors de ses frontières visibles par son Esprit. La révélation de Jésus-Christ constitue la thématisation parfaite de l'expérience de la grâce que tout homme peut mener ${ }^{4}$; de ce fait, l'homme qui assume l'autocommunication de Dieu devient non seulement un théiste anonyme, mais aussi un chrétien anonyme, sans qu'il soit, pour ainsi dire, dispensé du souci de connaître la vérité de l'Évangile, et sans réduire la signification de la mission, de l'évangélisation et des sacrements.

Les membres des autres traditions religieuses vivent un christianisme anonyme par la pratique de leurs traditions, où se trouvent des éléments de grâce, dit-on, fragmentaires, incomplets et même déficients. S’il en est ainsi, le christianisme aurait deux formes, à savoir implicite et explicite, lesquelles mettent l'homme en contact avec le mystère salvifique du Christ (Dupuis 2002, 93-965).

Nous avons abordé brièvement la théorie du chrétien anonyme, parce qu'elle est - à notre avis - la base de la théologie du pluralisme religieux représenté par des théologiens contemporains comme E. Schillebeeckx, C. Geffré et D. Tracy. Ceux-ci s'accordent, d'une part, sur la nécessité du

4. Rahner $(1965,549)$ : «Die ausdrücklich christliche Offenbarung wird zur reflexen Aussage der gnadenhaften Offenbarung, die der Mensch in der Tiefe seines Wesens schon immer unreflex erfährt ».

5. Dupuis distingue pourtant entre la position de Rahner et celle de Panikkar en observant chez Panikkar une distinction «entre le Christ mystère et le Jésus historique qui ne semble plus rendre suffisamment compte de l'affirmation chrétienne selon laquelle Jésus est Christ». La difficulté que Dupuis a avec la pensée de Panikkar concerne la non-identité entre le Christ et le Jésus historique, bien que cela ne fut pas la préoccupation de la communauté chrétienne primitive qui prêchait Jésus comme Christ ressuscité par le Père. C'est pourquoi Dupuis soutient l'inséparabilité de Jésus de l'histoire et du Christ de la foi. Le mystère du Christ dans la présentation de Rahner ayant une présence universelle tant dans le christianisme que dans les autres traditions religieuses est le mystère de la personne du Jésus historique. 
dépassement d'un certain ecclésiocentrisme qui radicalise la théologie barthienne faisant du christianisme l'unique religion de la grâce, et d'autre part à ne pas se contenter d'un certain inclusivisme christologique. Ces théologiens vont chercher à opérer ce tournant sans renoncer pour autant à la prétention légitime du christianisme comme «religion de la manifestation absolue et définitive de Dieu en Jésus-Christ» (Geffré 2006a, 83).

Pour Geffré, la théologie du XXe siècle a pris en considération l'horizon de l'athéisme et de la division de l'Église pour réinterpréter certains énoncés fondamentaux de la foi chrétienne. En revanche, ce XXI siècle présente un autre défi à la foi chrétienne: celui du pluralisme religieux, qui amène à une réinterprétation nouvelle du christianisme et entraîne des déplacements fondamentaux dans le traitement des questions dogmatiques. Le pluralisme religieux est en quelque sorte un nouveau signe des temps.

Si la tâche théologique se réclame d'une corrélation entre le message chrétien, les différentes cultures et la situation présente du monde, elle a, plus que jamais, l'urgence de relever le défi du pluralisme religieux. Pour ce faire, il lui est nécessaire de trouver un juste langage qui rende justice à la vérité chrétienne et qui entreprenne un dialogue franc et ouvert avec les autres traditions religieuses.

Certains théologiens vont jusqu'à adopter une position clairement pluraliste qui renonce à un christocentrisme inclusif au profit du théocentrisme $^{6}$ : ils considèrent alors toutes les religions comme gravitant autour de l'unique mystère de Dieu. Mais ce faisant, ils outrepassent la ligne de pensée théologique, telle qu'elle est rappelée par le récent document du Magistère romain Dominus Jesus (Congrégation pour la doctrine de la foi 2000). Sous prétexte que "Dieu seul sauve ", ils relativisent le salut offert en Jésus-Christ: «Le Christ serait bien une voie normative pour les chrétiens, mais il ne serait pas une voie exclusive de salut " (Geffré 2001b, $115^{7}$ ). Geffré, en se réclamant de la Wirkungsgeschichte de Gadamer, s'attache à la foi apostolique et à la tradition de l'Église: il ne se réclame donc

6. À ce propos, Geffré cite les théologiens suivant: Knitter (1981) et Haight (1999). Le principal instigateur d'une théologie pluraliste des religions fut le théologien britannique John Hick (1980, entre autres).

7. Toutefois, selon Geffré (2004, 39-62), Dominus Jesus ne réussit pas à concilier l'universalité du mystère du Christ avec la pleine reconnaissance de la valeur positive des religions non chrétiennes. Il s'agit d'un christocentrisme et d'un ecclésiocentrisme qui rappellent, d'une certaine façon, une théologie préconciliaire. 
pas d'un théocentrisme qui sacrifie le christocentrisme et la normativité de la christologie.

La théologie du pluralisme religieux, telle que la conçoit Geffré, entend dépasser la théologie de l'accomplissement qui ne respecte pas suffisamment l'altérité des autres traditions religieuses, mais sans compromettre l'unicité du mystère du Christ, autrement dit, « un christocentrisme constitutif» (Geffré 2001b, 1098). Aussi est-il possible de concilier un véritable pluralisme inclusif avec une christologie constitutive pour tous les hommes et pas seulement normative pour les seuls chrétiens. La théologie des religions ne doit donc pas sacrifier le christocentrisme à un théocentrisme indéterminé; sa tâche conduira plutôt à un approfondissement de la christologie qui n'absolutise pas le christianisme et qui respecte l'altérité des autres traditions religieuses (Geffré 2006b, 20). La fidélité de C. Geffré à l'héritage $\mathrm{du}$ Concile le mènera à proposer une approche relationnelle du christianisme, qui le désabsolutise et puisse l'ouvrir aux autres traditions religieuses.

\section{La kénose, fondement de la théologie du pluralisme religieux}

Le récit de la tour de Babel (Gn 11) témoigne d'une condamnation de l'homme qui voulait construire un royaume uniforme, de façon à se substituer à Dieu et supprimer la pluralité linguistique, culturelle et religieuse. La pluralité est chère à Dieu, et on en trouve la preuve dans les cultures de l'Ancien Testament ayant préparé la Révélation de Jésus-Christ, ainsi que dans la multiplicité culturelle qui s'est avérée nécessaire le jour de la Pentecôte, afin de traduire la richesse du Mystère de Dieu (Geffré 2006a, 63).

Qui dit pluralité des cultures dit aussi pluralité des religions et il n'est pas interdit de penser que la Pentecôte répond à Babel. Or, la Pentecôte apparaît précisément comme une sorte de légitimation qui est donnée à la pluralité, dans la mesure où la richesse surabondante du mystère de Dieu ne peut être exprimée que par une pluralité des formes religieuses (Geffré 2001a, 96).

La première observation que suscite la multiplicité religieuse, c'est son rapport avec la multiplicité des cultures. La religion est un produit de la culture, notamment parce qu'elle s'exprime dans un certain nombre de mythes et de rites. La Pentecôte témoigne de la richesse du mystère de Dieu qui, dans

8. Par christologie constitutive, Geffré veut dire qu'il est vrai que «Dieu seul sauve, mais c'est Jésus-Christ qui est l'actualisation même du vouloir divin quant au salut de l'homme» (voir Geffré 2004, 43). 
l'unicité du dessein divin, se manifeste dans une pluralité de formes religieuses. Il importe pourtant, selon Geffré, de ne pas confondre la pluralité des voies qui mènent vers Dieu avec le privilège unique du christianisme.

La religion se situe au cœur des cultures: aussi faut-il resituer le vocabulaire religieux dans son contexte culturel, pour être compréhensible. On désignera, par exemple, le bouddhisme comme "religion de salut", dans un sens de libération et de transformation de l'existence humaine (Geffré 2006a, 233). Par ailleurs, l'homme contemporain cherche un salut comme bonheur, guérison et délivrance, mais sans médiation d'un sauveur, tandis que les religions monothéistes entendent par salut - dont l'auteur est Dieu - la libération du péché et de la mort. S'il reste absolument vrai que la bonne nouvelle de Jésus-Christ est universelle, c'est-àdire transculturelle, elle n'est toutefois compréhensible que dans et par la médiation d'une culture donnée. Car si le message est universel, l'auditeur est toujours particulier et situé dans un contexte culturel et religieux déterminé.

La plus grande difficulté qu'affronte l'Église à l'heure de la mondialisation, c'est la rupture avec la culture occidentale dominante au profit d'un œcuménisme interreligieux qui correspond à l'unicité de la famille humaine (Geffré 2001a, 105 ${ }^{9}$ ). L'engouement pour le religieux qui coïncide avec un discrédit des idéologies désigne la phénoménologie du temps présent; nos contemporains posent des choix religieux détachés de leur culture d'origine et fusionnent ces choix avec leur propre culture.

Le grand défi actuel en Occident pour une ouverture à la Parole de Dieu, c'est cette mentalité plurielle incertaine - tant culturelle que religieuse - qui se traduit par une diversité d'options religieuses, éthiques, sociales et politiques. La «culture d'incertitude» coïncide avec une crise de la vérité manifestée dans le rejet que font certains chrétiens du Magistère de l'Église catholique, et l'orientation vers un syncrétisme religieux fournissant une expérience plus dynamique. Outre le pluralisme religieux, la proclamation de l'Évangile se heurte aux vieilles cultures du monde (Geffré 2006a, 337): il devient nécessaire d'aller au-delà des limites du christianisme passé - à savoir la culture d'Athènes et de Jérusalem —, pour viser une formulation du message chrétien conforme avec toutes les cultures du monde contemporain, auquel nous voulons annoncer l'Évangile.

9. En parlant d'œcuménisme interreligieux, Geffré souligne que "pour la première fois dans l'histoire, les hommes ont conscience de constituer une unique famille habitant dans la même maison commune». 
Face au défi du pluralisme, Geffré procède à une réinterprétation de la théologie de l'accomplissement dans un sens nouveau. Il prend en compte la dimension eschatologique du Règne de Dieu et tend à distinguer entre le Règne de Dieu et les formes historiques d'actualisation du christianisme (Cortesi 2006, 103). Pour autant, le théologien français prend ses distances par rapport à deux options théologiques que sont la théologie dialectique de Barth et le néolibéralisme qui sacrifie la norme christologique au profit du dialogue interreligieux (Geffré 2006a, 84).

L'enjeu consiste à maintenir une christologie inclusiviste constitutive qui ne sacrifie pas l'unicité et l'universalité du mystère du Christ, ou encore à dépasser le dilemme entre absolutisme et relativisme. En effet, il n'est pas nécessaire d'adopter une théologie pluraliste qui coïncide avec un théocentrisme indéterminé pour favoriser un vrai dialogue interreligieux. Il est possible de concilier une christologie constitutive et un pluralisme inclusif ${ }^{10}$. À cette fin, Geffré a recours à l'incarnation du Verbe de Dieu dans la personne historique de Jésus de Nazareth:

L'incarnation est la présence de l'Absolu de Dieu dans la particularité historique de Jésus de Nazareth, qui nous conduit à ne pas absolutiser le christianisme [...] les chrétiens dans l'Église ne sont les propriétaires ni de Dieu ni du salut. Ils sont seulement les témoins du Royaume de Dieu advenu en Jésus-Christ, tout en sachant que le Royaume advient dans les cœurs et dans l'histoire bien au-delà des frontières de l'Église ${ }^{11}$.

L'événement de la kénose a définitivement marqué l'histoire, et c'est dans l'incarnation que réside l'absolue singularité de Jésus-Christ. La

10. Concernant les expressions de christologie constitutive et de pluralisme inclusif, on renvoie à Geffré (2004, 43-46). L'expression pluralisme inclusif est d'abord employée par J. Dupuis et ensuite par C. Geffré afin de reconnaître la valeur irréductible des autres traditions religieuses. En d'autres termes, le théologien n'est pas condamné à choisir entre un inclusivisme christologique et un pluralisme qui coïnciderait nécessairement avec un théocentrisme indéterminé à la manière de John Hick. Et le maintien d'un christocentrisme constitutif dans le sens de l'unicité du salut réalisé en Jésus-Christ ne conduit nullement à un exclusivisme du christianisme ou de l'Église dans l'ordre du salut. En un mot, Geffré prend le juste milieu entre la théorie de l'accomplissement qui ne respecte pas l'altérité des autres religions et les théologiens pluralistes qui sacrifient le christocentrisme au prix du théocentrisme.

11. Geffré $(2006 a, 243)$; voir aussi Jean Paul II (1992, n 28): "l'Esprit est [...] à l'origine même de l'interrogation existentielle et religieuse de l'homme qui ne naît pas seulement de conditions contingentes mais aussi de la structure même de son être. La présence et l'activité de l'Esprit ne concernent pas seulement les individus, mais la société et l'histoire, les peuples, les cultures, les religions". 
kénose a inauguré un mode différent de penser et de parler de Dieu, en raison de la révélation faite par anéantissement. La kénose exprime d'une manière inédite l'autocommunication de Dieu à l'homme, et par là autorise le dépassement d'un certain dualisme entre Dieu et le monde, lequel rend peu intéressante la proposition chrétienne. Le paradoxe de la kénose opère un mouvement de crise et de rupture vis-à-vis d'une connaissance du Dieu naturel, parce qu'il met en évidence une différence qualitative de la Révélation. La kénose est dorénavant le critère de compréhension de la transcendance, en renvoyant à un Dieu qui pose son regard sur la souffrance des hommes avant d'imputer leurs péchés.

La compassion, christologiquement parlant, renvoie à une présence qui saurait laisser un espace à l'autre, jusqu'à supporter son éloignement, tel qu'il est advenu dans la crucifixion. Dans le Christ, Dieu se cache, en révélant sa condition existentielle, pour que soit donnée à chaque homme la liberté d'un choix, par lequel il vivra la radicalité de l'Évangile dans la construction du Royaume ${ }^{12}$.

Cette référence au théologien italien Carmelo Dotolo illustre bien la raison pour laquelle Geffré a fait le choix de la kénose comme point de départ de la théologie des religions. En fait, le paradoxe du christianisme se manifeste dans le mystère du Christ qui porte l'identité de l'absolument concret et de l'absolument universel (Geffré 2006a, 87; aussi Fruchon 2000, 165). L'ouverture du christianisme aux autres traditions religieuses s'enracine dans la théologie de la croix; c'est le mystère de la croix qui permet au christianisme d'être relationnel et dialogal. Le paradoxe de la kénose et de la foi nous introduit dans la logique de l'universel et du particulier de la figure de Jésus qui, mourant à sa particularité sur la croix, renaît à la figure d'universalité concrète du Christ. Geffré applique le modèle du Christ au christianisme afin que ce dernier se comprenne à la fois dans sa particularité historique et dans son universalité.

Le paradoxe du Logos fait chair scandalise les membres des autres religions, car il s'agit du paradoxe de la présence de Dieu et de son rapport avec l'événement particulier et contingent de Jésus-Christ. Geffré dénonce les tentatives de certains théologiens de réduire l'incarnation à une simple

12. Dotolo $(2007,221)$ : «la compassione, cristologicamente intesa, rinvia ad una presenza che sa fare spazio all'altro, fino a sopportarne l'allontanamento, come avvenuto nella crocifissione. In Cristo, Dio nasconde, rivelando, la sua condizione esistenziale, perché sia donata ad ogni uomo la libertà di una scelta capace di vivere la radicalità del Vangelo nella costruzione del Regno ». 
métaphore, ou de relâcher le lien indissoluble entre le Christ de la foi et le Jésus de l'histoire ${ }^{13}$. Ces tentatives compromettent en fait l'unicité du christianisme lorsqu'elles réduisent Jésus-Christ à une simple manifestation parmi d'autres dans l'histoire. Le paradoxe de l'incarnation n'est pas à prendre dans un sens mythique mais réaliste, en tant qu'un événement qui transcende toutes les possibilités humaines.

La singularité du christianisme, c'est qu'il témoigne de la révélation finale qui est inséparable du mystère de mort et de résurrection, et c'est cette continuité entre le mystère du Logos et sa pleine manifestation dans l'histoire en Jésus de Nazareth qui fait du christianisme une religion unique (Geffré 2006a, 90). Le mystère de la kénose du Fils de Dieu est la base de la résurrection et le fondement de l'Église. Aussi le mystère de la kénose apparaît-il comme la condition d'ouverture vers les autres — d'où l'absurdité de favoriser le dialogue interreligieux en évacuant une christologie normative au profit d'un théocentrisme. "Pourquoi [...] seul un théocentrisme radical répondrait-il aux exigences du dialogue interreligieux? Il semble qu'un approfondissement de la christologie ouvrirait des voies plus fécondes qui respecteraient à la fois les exigences d'un vrai pluralisme et celles de l'identité chrétienne» (Geffré 1992, 72).

Le refus de l'absoluité du christianisme et de l'unique médiation du Christ pour le salut se dresse comme un grand obstacle de rencontre entre les religions et le christianisme. Les religions monothéistes fournissent une expérience religieuse avec un Dieu personnel, alors que les religions asiatiques tendent à rencontrer le divin à l'intérieur de l'homme et du cosmos. Est-il pourtant acceptable de faire de la reconnaissance d'un Dieu unique le critère de cette rencontre? Geffré dénonce cette solution et recourt au principe de «la préoccupation ultime» (Geffré 2006a, 101). Il s'aperçoit que l'expérience religieuse authentique et l'expérience de la réalité ultime exprimée par les mystiques sont les points de rencontre par excellence entre toutes les religions ${ }^{14}$.

13. Concernant les théologiens qui réduisent l'incarnation à une simple métaphore, voir Hick (1973); et quant aux théologiens qui séparent entre le mystère du Christ et le Jésus historique, voir Panikkar (1972).

14. On renvoie à Holland $(2002,58)$, où Tracy joint Geffré quant à la préoccupation ultime comme point de rencontre entre les religions. Tracy s'aperçoit que l'Écriture Sainte finit avec la phrase "vient Seigneur Jésus ", c'est pourquoi il est opportun d'insérer un aspect apocalyptique en christologique. Tracy traite de la christologie dans un tiraillement entre le Christ qui est venu et qui viendra, en vue d'une interprétation théologique du christianisme en relation avec les autres religions. 
La kénose est donc un mystère qui donne à la croix de Jésus, dans sa particularité, une valeur universelle. L'attente chrétienne de la pleine réalisation du plan du salut à la fin de l'histoire fonde l'égalité du dialogue, sans atténuer pour autant l'intérêt ultime de la christologie (Fruchon 2000, 168). Il s'ensuit que le dialogue ne consiste plus à communiquer à l'autre la plénitude de la vérité contenue dans le christianisme, mais à être dans l'attente de la manifestation finale de la vérité universelle.

\section{Signification du pluralisme religieux dans le plan de Dieu}

Le rapport entre l'Église et les religions peut être abordé de deux manières: soit à partir du salut des non-chrétiens, soit à partir de l'être-sujet des autres. De là découle que le théologien cesse de considérer les membres des autres traditions religieuses comme des non-chrétiens, des pas-encorechrétiens ou des chrétiens anonymes; il les prend ainsi au sérieux comme des êtres devant Dieu (Waldenfels 1997, 632).

Ainsi, l'actuelle recherche ne se limite pas à scruter le problème du salut des membres des autres traditions religieuses, mais à chercher le dessein de Dieu pour le genre humain, la pluralité des fois vécues et des traditions religieuses dont nous sommes entourés, et la valeur des religions en tant que moyens pour entreprendre un rapport avec Dieu (Dupuis 1997, 22). Les théologiens posent la question de la signification de la pluralité des traditions religieuses dans le plan de Dieu pour l'humanité, ainsi que celle des racines du pluralisme répandu dans le monde.

La position prise dans cette recherche trouve son point de départ dans le deuxième Concile du Vatican, lequel favorise une attitude nouvelle des chrétiens et des Églises envers les membres d'autres religions, et apaise le contexte tendu qui marquait le rapport avec les religions (Dupuis 2002, 99). C'est pourquoi faire une théologie des religions à partir de la question du salut semble être inutile et ne répond guère au défi posé à la théologie chrétienne par la pluralité religieuse. Le Concile va au-delà du respect des membres des autres traditions religieuses, pour apprécier les valeurs positives de leurs religions comme une "préparation évangélique »:

L'Église catholique ne rejette rien de ce qui est vrai et saint dans ces religions et elle exhorte même ses fils pour que, avec prudence et charité [...] tout en témoignant de la foi et de la vie chrétienne, ils reconnaissent, préservent et fassent progresser les valeurs spirituelles, morales et socio-culturelles qui se trouvent en elles. (Nostra Aetate $\mathrm{n}^{\circ} 2$ ) 
Ce texte du Concile applique d'une certaine manière la théologie des semences $d u$ Verbe des Pères aux religions non chrétiennes. Néanmoins, pendant presque quarante ans, la théologie catholique a eu beaucoup de mal à prendre au sérieux les implications proprement théologiques de cette nouvelle attitude de l'Église. Geffré dit que ce jugement positif n'exprime pas suffisamment le rapport positif que les religions non chrétiennes peuvent avoir à l'Absolu et ne fournit pas une théologie des religions au sens strict du terme (Geffré 2001b, 106-107).

Geffré va donc effectuer un déplacement vers une théologie qui réfléchit sur la signification du pluralisme religieux, à l'intérieur du plan de Dieu, et qui s'interroge sur le rapport positif que les religions mondiales ont avec l'Absolu (Dupré La Tour 1999, 217). Pour mener à bien cette tâche, Geffré invite à abandonner un langage absolu et à adopter un langage relationnel, en vue d'une théologie des religions plus pertinente (Geffré 2006a, 30).

Le souci du théologien et les interrogations qu'il pose sur les valeurs positives des religions émanent de la volonté salvifique de Dieu lui-même, exprimée en 1 Tim 2,4: «Dieu veut que tous les hommes soient sauvés et parviennent à la connaissance de la vérité ». En dépit de la condamnation explicite, par la Déclaration Dominus Jesus, des théologiens qui distinguent entre un pluralisme de fait et un pluralisme de jure, Geffré tient à cette distinction dans la mesure où elle nous invite à dépasser un christocentrisme abstrait et naï ${ }^{15}$.

La vitalité des religions non chrétiennes est-elle due à l'aveuglement des hommes, au péché, à l'échec de la mission de l'Église ou bien à un vouloir mystérieux de Dieu (Geffré 2001a, 94) ? Quoique le Concile ne considère pas les autres religions comme des voies de salut et ne prononce pas un jugement sur la signification du pluralisme religieux, celles-ci ne pourraient être simplement la conséquence de l'aveuglement de l'humanité. Le Concile soutient que l'Église est perfection et accomplissement des germes

15. Il faut noter pourtant que Geffré s'oppose à la condamnation effectuée par la Déclaration Dominus Jesus au n ${ }^{\circ}$ 4: "La pérennité de l'annonce missionnaire de l'Église est aujourd'hui mise en péril par des théories relativistes, qui entendent justifier le pluralisme religieux, non seulement de facto mais aussi de jure (ou en tant que principe)». Geffré accepte personnellement comme une hypothèse théologique féconde la distinction entre un pluralisme religieux de fait et un pluralisme de principe sans renoncer aux vérités fondamentales qui sont énumérées au ${ }^{\circ} 4$. Il souligne que Dominus Jesus demeure prisonnière d'un dilemme quasi insurmontable entre absolutisme et relativisme. Voir Geffré (2004, 44; 2006b, 21). 
de grâce qui se trouvent en dehors du christianisme (AG 9): de là, la mission chrétienne consiste à découvrir les semences $d u$ Verbe cachées dans les traditions religieuses (AG 11).

L'Église garde, de quelque façon, une vision ecclésiocentrique qui débouche naturellement sur la théorie de l'accomplissement selon laquelle les autres religions atteignent leur perfection dans le christianisme. La théologie de l'accomplissement est conçue comme l'autocommunication de Dieu dans le Fils incarné Jésus-Christ, laquelle est la réponse définitive à la quête humaine de Dieu. La singularité du christianisme se manifeste dans l'incarnation dévoilant la recherche que Dieu fait de l'homme pour lui indiquer les voies pour l'atteindre (voir Jean Paul II 1996, n 6).

Le magistère de Jean Paul II va au-delà des affirmations conciliaires et soutient que l'Esprit Saint est présent et actif dans le monde, dans les nonchrétiens et dans leurs religions. "La présence et l'activité de l'Esprit ne concernent pas seulement les individus, mais la société et l'histoire, les peuples, les cultures, les religions » (Jean Paul II 1992, n² 28). Toutefois, c'est dans le document Dialogue et annonce qu'on note un vrai affranchissement de la théologie de l'accomplissement traditionnel, où est préconisée une présence active du mystère du Christ dans les traditions religieuses.

Il découle de ce mystère d'unité que tous ceux et celles qui sont sauvés participent, bien que différemment, au même mystère de salut en Jésus Christ par son Esprit [...] Concrètement, c'est dans la pratique sincère de ce qui est bon dans leurs traditions religieuses et en suivant les directives de leur conscience, que les membres des autres religions répondent positivement à l'appel de Dieu et reçoivent le salut en Jésus Christ, même s'ils ne le reconnaissent et ne le confessent pas comme leur Sauveur (cf. Ad gentes, 3, 9, 11) ${ }^{16}$.

Le pluralisme peut être un dessein divin dont la signification dernière nous échappe, parce que l'Esprit Saint offre à tous les hommes la possibilité de participer au mystère pascal du Christ «d'une façon que Dieu connaît»(GS 22). Ainsi les membres des autres traditions religieuses répondent à l'appel de Dieu par leur rectitude de vie, et ainsi ils reçoivent le salut du Christ. Les religions sont considérées dans le document cité ci-dessus comme une forme d'option anonyme pour le Christ, sans sacrifier pour autant le privilège unique de la révélation judéo-chrétienne. L'histoire du monde tout entière est sous la conduite de Dieu qui veut le salut et le bonheur des hommes qui peuvent être sauvés chaque fois qu'ils décident de travailler pour «la construction du Royaume de Dieu comme royaume de

16. Conseil pontifical pour le dialogue interreligieux (1991, $\left.\mathrm{n}^{\circ} 29\right)$. 
justice et de paix» (Geffré 2006a, 62). Il ne faut pas perdre de vue que le Verbe fait chair est venu au monde pour illuminer tout être humain, quelle que soit son appartenance religieuse ou sa culture.

L'expérience de la grâce n'est pas exclusivement chrétienne, l'expérience vécue dans les religions non chrétiennes favorise l'ouverture de l'homme à l'Absolu. Et c'est en fait la conclusion de la théologie des semences $d u$ Verbe appliquée aux religions mondiales, celles-ci se reflétant dans les dispositions subjectives des membres des autres religions, aussi bien que dans les rites et les coutumes des peuples (voir LG 17 et Redemptoris missio 56). Geffré en vient à interpréter les semences du Verbe comme des paroles de Dieu lui-même adressées aux hommes; de là découle que la plénitude de la révélation du Christ ne devrait pas exclure la possibilité que Dieu parle dans les autres traditions religieuses et qu'il se manifeste dans l'histoire en dehors du christianisme.

Cette théologie située dans l'horizon interreligieux va avoir une influence sur la christologie, car de fait les révélations que les religions contiennent sont liées nécessairement au Christ. La tâche que Geffré confie à la théologie interreligieuse ne consiste pas seulement à chercher «le Dieu inconnu », mais aussi «le Christ inconnu qui nous attend dans toute religion, voire dans tout être humain» (Geffré 2006a, 32). Il est évident que Geffré cherche à partir de la manifestation de Dieu en Jésus de Nazareth pour trouver la marque du caractère dialogal du christianisme.

Geffré va au-delà de la théologie de l'accomplissement en appréciant les traditions religieuses comme une œuvre suscitée par l'Esprit de Dieu, quoique le Christ demeure la récapitulation de toute l'histoire. S'il en est ainsi, l'Église doit changer l'axe de sa mission: tout en reconnaissant la nécessité de l'annonce du Royaume de Dieu, l'évangélisation ne devrait plus viser à convertir à tout prix les membres des autres traditions, mais «à les convoquer à l'inconditionnalité de la révélation finale » (Geffré 2006a, 38). Par conséquent, la responsabilité de la théologie dans le monde du pluralisme religieux consiste à promouvoir un christianisme mondial et polycentrique qui puise à la fois dans les traditions chrétiennes et dans les richesses des diverses cultures.

L'Église devrait "mourir » à sa figure particulière et achevée pour renaître à une plus grande universalité qui outrepasse ses déterminations historiques et spatiales; la théologie est également appelée à accueillir sa transformation prolongée dans le mystère de la kénose de Dieu. L'universalisme a abouti à son apogée dans la personne de Jésus-Christ, la Révélation définitive en personne; il nous rappelle l'alliance universelle que Dieu a conclue, 
déjà avec Noé: «jamais plus ». D’après Geffré, les religions peuvent médiatiser le salut comme signes imparfaits du salut accompli, réalisé par et dans le Christ. Si l'histoire du salut est l'histoire du Royaume de Dieu, les autres traditions religieuses appartiennent à l'histoire universelle du salut qui trouve son vrai sens et sa réalisation dans l'histoire du salut réalisée en Jésus-Christ, et dans l'Église son corps (Dhavamony 2004, 66).

Appelant à dépasser les frontières de l'Occident (Athènes et Jérusalem) dans l'approche théologique, la rencontre des religions ne portera plus sur les différences de doctrine entre les chrétiens et les non-chrétiens, mais à redécouvrir l'expérience commune de la Divinité. Si l'alliance avec Noé est cosmique et irrévocable, les grandes religions mondiales participent au salut, en tant que voie en quête de perfectionnement. L'irrévocable élection d'Israël et l'identité du dessein bienveillant seront les postulats et la clé interprétative de la théologie du pluralisme religieux qui veut aller au-delà de la théologie de l'accomplissement et reconnaître que l'Église ne remplace par les richesses des autres traditions religieuses (Aebischer-Crettol 2001, 733).

La révélation de Jésus-Christ est le témoignage par excellence de l'autocommunication de Dieu à l'humanité dans la grâce (voir Rahner - Ratzinger 1972, 15-36). Ainsi, dans la veine rahnérienne, Geffré affirme l'unicité de la révélation divine, dans sa coïncidence avec l'expérience spirituelle de l'Absolu que tout homme pourrait faire. Tel est le sens du discours de Paul aux Athéniens "ce Dieu que vous adorez, c'est celui-là même que je vous annonce» (Ac 17,23). "La prise au sérieux de l'humanité de Jésus nous invite à respecter la diversité des religions comme lieux d'une possible manifestation des richesses du mystère de Dieu» (Geffré 2006a, 67).

Geffré assume ainsi la position de Pannenberg sur l'histoire comme histoire du salut, parce que Dieu est en quête de l'homme depuis toujours, et que dans le même temps, l'homme est à recherche du Dieu inconnu. Les valeurs salutaires des autres religions dépendent fondamentalement du mystère du Christ, et constituent en quelque sorte une préparation évangélique selon l'expression propre à la théologie de l'accomplissement. Toutefois, la théologie de l'accomplissement implique une dépréciation des autres traditions religieuses en tant qu'elle considère leurs valeurs positives implicitement chrétiennes.

Dieu veut écrire une histoire du salut qui tende vers la plénitude du Royaume de Dieu à partir de l'histoire tout court, même si les hommes n'en ont pas conscience (Geffré 2006a, 72). Si Dieu veut s'autocommuniquer à l'homme, l'obéissance humaine est le chemin de l'accueil de ce don que Dieu fait de lui-même. Les membres des autres traditions religieuses 
appartiennent au Royaume de Dieu tant qu'ils répondent à l'appel de Dieu à travers leur conscience. Être membre du Royaume de Dieu consiste à promouvoir les valeurs du Royaume sans appartenir nécessairement à l’Église; la réponse à l'appel de Dieu signifie déjà être ordonné vers l'Église et sauvé en Jésus-Christ. À ce stade, tous les peuples de la terre sont appelés à vivre les valeurs évangéliques et à s'ouvrir à l'action de l'Esprit pour construire avec les chrétiens l'unique Royaume de Dieu.

Les valeurs implicitement chrétiennes présentes dans les autres religions sont en fait des valeurs christiques, liées au mystère du Christ. Par conséquent, Geffré renonce à l'expression "valeurs chrétiennes» en faveur de «valeurs christiques": "On peut entendre par là des germes de vérité, de bonté et de sainteté qui ont un lien secret avec la christianité, c'est-à-dire le potentiel christique de tout être humain qui a été créé non seulement à l'image de Dieu mais à l'image du Christ nouvel Adam» (Geffré 2008, 31-41).

\section{Conclusion}

Il s'avère que Geffré tend, dans sa théologie du pluralisme religieux, à dépasser la théologie de l'accomplissement soutenue par le Vatican II. En plus, malgré la tension qui marque certaines positions de Geffré avec le Magistère actuel, le théologien français reste fidèle à la voie christologique en refusant catégoriquement de sacrifier l'unicité et l'universalité de JésusChrist dans son dialogue avec les autres traditions religieuses.

En s'ouvrant vers les autres religions, l'Église doit trouver un nouveau style de présence au monde, selon lequel le christianisme se présente comme la religion de l'Évangile et de «l'humain authentique» (Geffré 2009, 583). L'orientation de Geffré revendique une nouvelle orientation théologique qui favorise l'avènement d'un christianisme polycentrique faisant face à un monde unidimensionnel. L'œcuménisme interreligieux ne devrait certainement pas conduire à l'indifférentisme mais à une meilleure compréhension de l'identité chrétienne (Geffré 2006a, 31777).

17. On renvoie ici à l'article récent de Geffré (2009, 579), où en se rendant compte de l'échec du catholicisme institutionnel à imposer ses valeurs à la société sécularisée, il souligne que la tradition évangélique du christianisme peut résister aux défis de la société contemporaine et doit rester une vocation mondiale allant au-delà des frontières de l'Occident. Il faut pourtant que cette vocation évangélique poursuive la démarche entreprise par le Concile Vatican II qui avait témoigné d'une avancée spectaculaire dans le domaine du dialogue avec la modernité, de l'œecuménisme et du dialogue interreligieux. 
Enfin, la rencontre entre le christianisme et les traditions religieuses entraîne une figure inédite du christianisme due à l'intégration d'éléments positifs des autres religions. La nouvelle vocation du christianisme, au sein de la diversité culturelle et religieuse, c'est d'assumer des attitudes spirituelles relatives à d'autres traditions religieuses du monde en les transfigurant selon la forme chrétienne, tout en maintenant Jésus-Christ comme son centre absolu.

\section{Références}

Aebischer-CretTol, M. (2001), Vers un œecuménisme interreligieux, Paris, Cerf.

CONGAR, Y. (2000) [1959], Vaste monde ma paroisse. Vérité et dimensions $d u$ salut, Paris, Cerf.

CONGRÉGATION POUR LA DOCTRINE DE LA FOI (2000), Déclaration «Dominus Iesus» : sur l'unicité et l'universalité salvifique de Jésus-Christ et de l'Église, Rome.

CONSEIL PONTIFICAL POUR LE DiALOGUE INTERRELIGIEUX (1991), Dialogue et annonce: Réflexions et orientations concernant le dialogue interreligieux et l'annonce de l'Évangile de Jésus-Christ, Cité du Vatican.

CORTESI, A. (2006), "Mistica, politica e dialogo interreligioso nella teologia di Claude Geffré », Etica \& Politica, 8, p. 89-115.

(2007), «De l'herméneutique théologique à la théologie interreligieuse ", Revue des sciences philosophiques et théologiques, 91, p. 285311.

DANIÉLOU, J. (1948), Le mystère du salut des nations, Paris, Seuil. (1953), Essais sur le mystère de l'histoire, Paris, Seuil.

Dhavamony, M. (2004), World Religions in the History of Salvation, Quincy, Franciscan.

Dotolo, C. (2007), Un cristianesimo possibile. Tra postmodernità e ricerca religiosa, Brescia, Queriniana.

Dupré La Tour, A. (1999), "Théologie des religions non chrétiennes et l'Islam ", Studia missionalia, 48, p. 215-230.

DupuIs, J. (1997), Vers une théologie chrétienne du pluralisme religieux, Paris, Cerf.

(2002), La rencontre du christianisme et des religions, Paris, Cerf. 
FRUCHON, P. (2000), «Sur une théologie chrétienne du pluralisme religieux. Statut d'un dialogue ", Science et Esprit, 52, p. 159-169.

GADAMER, H.-G. (1986) [1960], Wabrheit und Methode: Grundzüge einer philosophischen Hermeneutik, Tübingen, Mohr.

GEFFRÉ, C. (1992), «Théologie chrétienne et dialogue interreligieux », Revue de l'Institut Catholique de Paris, 38/1, p. 63-82.

(1999), Profession théologien, quelle pensée chrétienne pour le $X X I^{e}$ siècle?, Entretiens avec Gwendoline Jarczyk, Paris, Albin Michel. (2000), "Le pluralisme religieux et l'indifférentisme ou le vrai défi de la théologie chrétienne ", Revue Théologique de Louvain, 31, p. 3-32. (2001a), Croire et interpréter, Paris, Cerf.

(2001b), «La théologie des religions ou le salut d'une humanité plurielle», Raisons politiques, 4, p. 104-120.

(2001c), "Le tournant herméneutique de la théologie", Transversalités. Revue de l'Institut Catholique de Paris, 80, p. 189-202.

(2004), «Le pluralisme religieux comme nouveau paradigme de la théologie ", dans M. Dumas, F. Nault, L. Pelletier, dir., Théologie et culture: hommages à Jean Richard, Québec, Presses de l'Université Laval, p. 39-62. Paris, Cerf.

(2006b), «Profession théologien, retour sur plus de quarante ans de pratique ", Laval théologique et philosophique, 62, 7-21.

(2008), «Les richesses spirituelles des autres religions », Cahiers de saint Dominique, 293, 31-41.

(2009), «L'avenir du christianisme face au défi du pluralisme culturel et religieux ", Revue de Sciences Religieuses, 4, p. 567-585.

GibelLinI, R. (2008), Breve storia della teologia del XX secolo, Brescia, Morcelliana.

GRondin, J. (2005), "La fusion des horizons. La version gadamérienne de l'adaequatio rei et intellectus?", Archives de philosophie, 68, 401-418.

Guggenheim, A. (2006), "La théologie de l'accomplissement de Jean Daniélou ", Nouvelle revue théologique, 128, p. 240-257.

HaIght, R. (1999), Jesus as Symbol of God, Maryknoll, Orbis Books. Hick, J. (1973), God and the Universe of Faiths, New York, Macmillan. 
(1980), God Has Many Names, Philadelphia, Westminster Press.

Holland, S. (2002), "The Side of God: A Conversation with David Tracy ", Crosscurrents, 52, p. 54-59.

Jean Paul II (1992), La mission du Christ Rédempteur, Paris, Cerf.

(1996), Tertio Millennio adveniente: Commentaire théologique et pastoral, Paris, Mame.

KNITTER, P. (1981), No Other Name? A Critical Survey of Christian Attitudes toward World Religions, Maryknoll, Orbis Books.

Lubac De, H. (1946), Le fondement théologique des missions, Paris, Seuil. (1965), Le mystère du surnaturel, Paris, Aubier

PanikKaR, R. (1972), Le Christ et l'hindouisme, Paris, Centurion.

RAHNER, K. (1965), Schriften zur Theologie VI, Zürich, Einsiedeln.

(1983), Traité fondamental de la foi. Introduction au concept de christianisme, Paris, Centurion.

RATZINGER J. (1972), Révélation et tradition, Paris, Desclée de Brouwer.

(1983) [allemand 1976], Traité fondamental de la foi, Paris, Centurion.

RichARD, J. (2002), "Thèses pour une théologie pluraliste des religions", Laval théologique et philosophique, 58, p. 27-42.

(2006), «Théologie herméneutique et théologie interreligieuse: à propos de Croire et Interpréter", Laval théologique et philosophique, 62, p. 23-34.

Salman, W. (2010), "Conséquences du tournant herméneutique de Claude Geffré ", Transversalités. Revue de l'Institut Catholique de Paris, 113, p. $137-152$.

SesBoüé, B. (1984), «Karl Rahner et les chrétiens anonymes », Études, 361, p. 521-535.

Waldenfels, H. (1997), Manuel de théologie fondamentale, Paris, Cerf. (1999), «Unterwegs zu einer christlichen Theologie des religiösen Pluralismus. Anmerkungen zum Fall Dupuis », Stimmen der Zeit, 217, p. 597-610. 


\section{Résumé}

L'article expose la théologie des religions de Claude Geffré en la situant dans son contexte de théologie catholique des religions. De Babel à Pentecôte est l'ouvrage dans lequel le théologien français se rend compte de la situation pluraliste du monde. Il s'agit d'une approche théologique qui propose des solutions et fait une lecture créative de la tradition chrétienne, notamment de celle des semences $d u$ Verbe. Nous retenons certains points essentiels de la théologie de Geffré: le caractère kénotique du christianisme et la réflexion sur la signification du pluralisme.

\section{Abstract}

This article sets forth Claude Geffrés theology of religions and situates it in the wider context of the Catholic theology of religions. In De Babel à Pentecôte, Geffré shows his awareness of a world characterized by religious pluralism, suggesting some solutions. He sheds a new light on Tradition, especially that of the seeds of the Logos. This article especially emphasizes some key points of Geffrés theology: the kenotic nature of Christianity and his reflection on the meaning of religious pluralism. 